\title{
BMJ Open Long-term outcome of therapist-guided internet-based cognitive behavioural therapy for body dysmorphic disorder (BDD-NET): a naturalistic 2-year follow- up after a randomised controlled trial
}

\author{
Jesper Enander, ${ }^{1}$ Brjánn Ljótsson, ${ }^{1,2}$ Lina Anderhell, ${ }^{2}$ Martin Runeborg, ${ }^{2}$ \\ Oskar Flygare, ${ }^{2}$ Oskar Cottman, ${ }^{2}$ Erik Andersson,, ${ }^{1,2}$ Sofia Dahlén, ${ }^{3}$ \\ Linn Lichtenstein, ${ }^{3}$ Volen Z Ivanov, ${ }^{1,3}$ David Mataix-Cols, ${ }^{1,3}$ Christian Rück ${ }^{1,3}$
}

To cite: Enander J, Ljótsson B, Anderhell L, et al. Long-term outcome of therapist-guided internet-based cognitive behavioural therapy for body dysmorphic disorder (BDDNET): a naturalistic 2-year follow-up after a randomised controlled trial. BMJ Open 2019;9:e024307. doi:10.1136/ bmjopen-2018-024307

- Prepublication history for this paper is available online. To view these files please visit the journal online (http://dx.doi org/10.1136/bmjopen-2018024307).

Received 20 May 2018 Revised 25 July 2018 Accepted 11 October 2018

\section{Check for updates}

(c) Author(s) (or their employer(s)) 2019. Re-use permitted under CC BY-NC. No commercial re-use. See rights and permissions. Published by BMJ.

${ }^{1}$ Centre for Psychiatry Research, Department of Clinical Neuroscience, Karolinska Institutet, Stockholm, Sweden ${ }^{2}$ Division of Psychology, Department of Clinical Neuroscience, Karolinska Institutet, Stockholm, Sweden ${ }^{3}$ Stockholm Health Care Services, Stockholm County Council, Stockholm, Sweden

Correspondence to Dr Jesper Enander; jesper.enander@ki.se

\section{ABSTRACT}

Objectives Most patients with body dysmorphic disorder (BDD) do not receive evidence-based treatment. A randomised controlled trial (RCT) has found that a therapist-guided internet-based cognitive-behavioural therapy (CBT) programme for BDD (BDD-NET) can be delivered safely via the internet with significant improvements in BDD symptom severity in the short term. The purpose of this study was to evaluate if the therapeutic gains of BDD-NET are maintained 2 years after treatment.

Setting Academic medical centre.

Participants A naturalistic 2-year follow-up study of the 88 self-referred adult outpatients with a diagnosis of BDD that had received BDD-NET within the context of the RCT.

Primary and secondary outcomes The primary outcome was the BDD-Yale-Brown Obsessive-Compulsive Scale (YBOCS). Responder status was defined as a $\geq 30 \%$ reduction in symptoms. Remission was defined as no longer meeting Diagnostic and Statistical Manual of Mental Disorders 5th edition criteria for BDD. Secondary outcomes included measures of depression, global functioning and quality of life.

Results The efficacy of BDD-NET was sustained long- term, with further improvements observed on the BDD-YBOCS during the follow-up period. At follow-up, 69\% (95\% Cl 57\% to $80 \%)$ were classified as responders and $56 \%(95 \% \mathrm{Cl} 43 \%$ to $69 \%$ ) were in remission. Gains on depressive symptoms and global functioning were also sustained but not quality of life. A majority of participants reported that the main reason for seeking help for their BDD was the possibility to access the treatment from home.

Conclusion BDD-NET is an effective treatment for BDD, and the patients' gains are maintained in the long term. BDD-NET has the potential to increase access to CBT and may lower the threshold for BDD sufferers to seek help in the first place.

Trial registration number NCT02010619.

\section{INTRODUCTION}

Body dysmorphic disorder (BDD) is characterised by a preoccupation with perceived
Strengths and limitations of this study

- This study is the first to explore the long-term outcomes of a novel therapist-guided, internet-based programme designed to increase access to cognitive-behavioural therapy for patients with body dysmorphic disorder.

- Missing data were carefully imputed using multiple imputations; however, the estimates should be interpreted with caution.

- All participants were self-referred and hence particularly motivated for treatment.

- The uncontrolled nature of the study limits the possibility to make causal interferences as to what caused the further improvements observed during the follow-up.

defects in physical appearance that are not visible or only appear slight to others. ${ }^{1}$ Another hallmark of BDD is time-consuming repetitive behaviours, such as mirror gazing or camouflaging of disliked body areas. ${ }^{1}$ Although often underdiagnosed within mental health services, ${ }^{2}$ BDD is a relatively common disorder with a reported prevalence of $0.7 \%-2.2 \%$ in the general population. ${ }^{3}$ BDD usually begins during adolescence with a reported mean age at onset of 16 years ${ }^{45}$ and is associated with functional impairment across various life domains, marked suicidality, high rates of psychiatric hospitalisation and reported to be relatively chronic if left untreated. ${ }^{1}{ }^{6-8}$ The National Institute for Health and Clinical Excellence recommends that sufferers of BDD should be offered either a course of a selective serotonin response inhibitor or specialised cognitivebehavioural therapy (CBT) ${ }^{9}$ In general, most people favour psychological interventions 
over medication, ${ }^{10}$ and two meta-analyses have shown that CBT is an effective treatment for BDD in the short term (2-4 months after treatment). ${ }^{11}{ }^{12}$ However, reports on the long-term outcomes of CBT for BDD are rare, with only three studies published with at least 12-month follow-up data. The first study followed up 10 patients who received an intensive treatment programme for BDD consisting of exposure and response prevention, 5 days per week for 6 weeks, with half of the participants randomised to a maintenance programme consisting of biweekly contacts for 6 months. Participants were able to maintain their treatment gains for up to 2 years after completing the programme. ${ }^{13}$ In the second study, 39 participants were followed up between 1 year and 4 years after they had participated in a randomised controlled trial (RCT) where they were given weekly sessions of CBT for up to 16 weeks. ${ }^{14}$ The participants who made significant gains during the acute treatment phase generally maintained their gains during the follow-up period. In total, $46 \%$ of the participants were responders at follow-up, and relapse rates were relatively low. ${ }^{14}$ In the third study, a group of adolescents who had originally participated in a RCT of developmentally tailored CBT for BDD ( $\mathrm{n}=26)$ were followed for up to 12 months after the end of treatment. ${ }^{15}$ BDD symptoms decreased significantly from baseline to post-treatment and remained stable over the 12-month follow-up. At this time point, $50 \%$ of participants were classified as responders and $23 \%$ as being in remission. ${ }^{15}$

Although CBT for BDD seems promising, many sufferers do not get access to this treatment due to factors such as a lack of trained therapists, costs associated with treatment and geographical barriers where certain areas are especially underserved. ${ }^{16-18}$ In two prospective naturalistic follow-up studies of people with BDD stretching over a period of $1-4$ years, only $10 \%-22 \%$ had received CBT for their BDD. ${ }^{6} 19$ The shortage of trained CBT therapists calls for new and alternative ways of delivering evidence based psychological treatments. ${ }^{20}$ Internet-based CBT has been shown to be effective for a wide range of psychiatric disorders, and cost-effectiveness research shows that this treatment modality, in general, has a high probability of being cost-effective when compared with no treatment or conventional CBT. ${ }^{20-22}$ Thus, internet-based CBT may play an important role in increasing access to effective treatment. With the aim to increase access to evidence-based care for BDD, we recently developed a therapist-guided internet-based CBT programme for BDD called BDD-NET ${ }^{23}$ In a RCT, BDD-NET was associated with significant improvements in BDD symptom severity, depression and other secondary outcomes in the short term. ${ }^{24}$ Overall, the short-term treatment effects of BDD-NET are similar to those gained in traditional faceto-face CBT, ${ }^{12}$ and it has the potential to greatly increase access to evidence-based psychiatric care for this common disorder. Whether the gains obtained with this form of treatment delivery are maintained long term is a critical yet unanswered question.
In order to determine the long-term treatment effects of BDD-NET on BDD symptoms, depression, general function and quality of life, we conducted a naturalistic 2-year follow-up of the participants in the original RCT that had received internet-based $\mathrm{CBT}^{24}$ Because many BDD sufferers report that important reasons for not seeking treatment include feelings of shame and fear of stigma associated with openly discussing their appearance concerns, ${ }^{1718}$ we also explored the participants' treatment preferences and reasons for seeking participation in the trial.

\section{METHOD \\ Design}

This was a naturalistic long-term follow-up study of all participants that had received internet-based CBT in a previously reported single-blind, parallel-group, superiority trial where participants $(n=94)$ were initially randomised to either therapist-guided internet-based CBT (BDD-NET; $\mathrm{n}=47$ ) or online supportive therapy $(\mathrm{n}=47)$ for 12 weeks. All participants $(n=47)$ who had initially been randomised to online supportive therapy were offered to cross over to BDD-NET at the 3-month follow-up point (6 months from baseline). Two participants were lost to follow-up, and four participants declined to be crossed over. In total, 88 participants out of the original sample of 94 received internet-based CBT and were included in the long-term follow-up. For a detailed description of recruitment, participants and procedure of the RCT, please refer to the original paper. ${ }^{24}$

\section{Inclusion and exclusion criteria}

All participants in the original trial had a principal diagnosis of BDD according to the Diagnostic and Statistical Manual of Mental Disorders (DSM-5) ${ }^{1}$ and a score of at least 20 on the modified Yale-Brown Obsessive-Compulsive Scale (BDD-YBOCS), corresponding to moderate BDD. ${ }^{25}$ Exclusion criteria in the RCT were psychotropic medication changes within 2 months prior to enrolment, completed CBT for BDD within the last 12 months, current substance dependence, bipolar disorder or psychosis, acute suicidal ideation, a severe personality disorder that could jeopardise treatment participation (eg, borderline personality disorder with self-harm) and concurrent psychological treatment.

\section{Intervention}

BDD-NET is delivered via a tailored online platform using a dedicated hospital server with encrypted traffic and an authentication login function to guarantee participant confidentiality. Treatment length is 12 weeks, and none of the participants has any face-to-face contact with a therapist. BDD-NET consists of self-help texts and worksheets that are delivered in eight interactive modules, each devoted to a certain theme. The modules are: (1) psychoeducation, (2) a cognitive behaviour conceptualisation of BDD, (3) cognitive restructuring, (4) exposure 
and response prevention, (5) more on exposure and response prevention, (6) values-based behaviour change, (7) difficulties encountered during treatment and (8) relapse prevention. The first five modules contain the core treatment components (ie, psychoeducation, cognitive restructuring, exposure and response prevention). Throughout the treatment, the participant has unlimited access to an identified therapist that may be contacted at any time through the platform's built in message system. In total, four therapists guided the participants through the treatment with patients equally split among them. The duration of therapist contact and sent messages was automatically recorded by the BDD-NET platform. Median therapist time spent weekly per participant reading and answering messages was $13.2 \mathrm{~min}$. In total, therapist sent an average of $25.0(\mathrm{SD}=24.2)$ messages during treatment. The role of the therapist is mainly to guide and coach the participant throughout the treatment, provide feedback on homework assignments, answer questions, provide support and consecutively grant access to the next treatment module. In essence, the treatment mimics that of face-to-face CBT but differs by the mode of delivery. The BDD-NET treatment protocol has been validated in a pilot trial and shown to be efficacious in a RCT. ${ }^{23} 24$

\section{Assessment points and outcomes}

All participants were assessed at baseline, immediately after treatment (post-treatment), 3 months after treatment (3-month follow-up), 1 year after treatment (12-month follow-up) and 2 years after treatment (24-month follow-up). After the 3-month follow-up, all patients first randomised to online supportive therapy were offered to crossover to receive BDD-NET. At all time points, participants underwent a formal structured diagnostic interview. The interviews were conducted over telephone, which has shown to be a reliable administration format for structured psychiatric assessments. ${ }^{26}$ To establish a diagnosis of BDD, the Structured Clinical Interview for Diagnostic and Statistical Manual of Mental Disorders IV (DSM-IV) Axis I Disorders was used, with an added question about the presence of repetitive behaviours to reflect the updates made to the diagnostic criteria of BDD in DSM-5. The Mini-International Neuropsychiatric Interview was used to determine the presence of other comorbid psychiatric disorders. ${ }^{27}$ All assessors had received extensive training in structured diagnostic interviews. Participants also completed online self-report measures at these time points.

The primary outcome was BDD symptom severity assessed with the clinician-administered BDD-YBOCS, ${ }^{25}$ rated by blinded assessors up until the control group crossed over to internet-based CBT. Blinding was then broken per protocol in the original RCT. The BDD-YBOCS is the gold standard for assessing BDD symptom severity and has a total score of $0-48$, with a higher score indicating more severe symptoms. To ensure quality of assessment with BDD-YBOCS, clinicians in this trial practised together on case examples with excellent reliability between raters (intraclass correlation 0.95 , $95 \%$ CI 0.89 to 0.98 ). Responder status was defined as an empirically derived cut-off point of at least a $30 \%$ reduction from baseline on the BDD-YBOCS. ${ }^{28}$ Remission was defined as no longer meeting DSM-5 diagnostic criteria for BDD. Secondary outcomes were depressive symptoms assessed with the Montgomery-Åsberg Depression Rating Scale Self-Rated (MADRS-S), ${ }^{29}$ clinician rated global functioning assessed with the Global Assessment of Functioning scale $(\mathrm{GAF})^{30}$ and health related quality of life was assessed with the self-rated EQ5D EuroQol (EQ5D). ${ }^{31}$

We also recorded if the participants had received any further psychological or pharmacological treatment at each single follow-up point. All participants completed a form regarding additional treatment: one section was devoted to psychological treatment and one section to pharmacological treatment. Participants were also asked about cosmetic treatment, such as plastic surgery, since it is common for patients with BDD to try and 'fix' perceived defects.

As many BDD sufferers report that important reasons for not seeking treatment include feelings of shame and fear of stigma associated with openly discussing their appearance concerns, ${ }^{17} 18$ participants completed a short online survey at the post-treatment assessment about past experiences with health services. In addition, they were briefly interviewed at the 12-month follow-up about their reasons for participating in the trial in the first instance (this was a post hoc research question).

\section{Patient and public involvement}

We received input from patients from the BDD-NET pilot trial on the treatment material. No patients were involved in setting the research questions or the outcome measures, nor were they involved in developing plans for recruitment, design or implementation of the study. No patients were asked to advise on interpretation or writing up of results. We carefully assessed the burden of the trial interventions on the patients by collecting information about adverse events, quality of life and time spent on the treatment. The results from the RCT has been disseminated to study participants and the Swedish OCD Foundation.

\section{Statistical analysis}

All analyses of primary and secondary continuous outcomes were done according to intention-to-treat principles, including the full sample of 88 participants. Linear mixed models (LMMs) were used to evaluate the effect of time on the different outcomes. LMMs take into account the interindividual differences in intraindividual change, and participant varying intercepts were included as a random effect in the model. ${ }^{32}$ Missing data were imputed using multiple imputations by chained equations. ${ }^{33}$ We carefully examined the missing data patterns and auxiliary variables that were correlated with missing data were included in the multiple imputation model to increase the precision of the estimates, ${ }^{34}$ such as demographic 
variables and baseline outcomes. All estimates with SEs were pooled from 50 imputations using 'Rubin's rules'. ${ }^{35}$ Multiple imputation has the potential to reduce bias and improves parameter estimates when missing data are due to dropouts in repeated measures designs, compared with complete-case analyses. ${ }^{36}$ Moreover, in data simulation models, the use of multiple imputation has shown to perform well even with $50 \%$ of missing observations. ${ }^{38}$ Responder status (at least a $30 \%$ reduction on the BDD-YBOCS) was calculated using data derived from the multiple imputations. Categorical data were analysed using $\chi^{2}$ tests, and independent $t$-tests were used for assessing between-group differences. Within-group effect sizes were calculated for observed data using Cohen's $d$. Statistical analyses were done in R (cran.r-project.org).

\section{RESULTS}

Figure 1 shows the flow of participants through the longterm follow-up. Eighty-eight participants $(100 \%)$ provided data at baseline, $81(92 \%)$ completed clinician-administered assessments at post-treatment, $75(85 \%)$ at 3-month follow-up, $54(61 \%)$ at 12-month follow-up and $56(64 \%)$ at 24-month follow-up. Online self-report measures were completed by 77 participants $(88 \%)$ at post-treatment, $65(74 \%)$ at 3-month follow-up, $37(42 \%)$ at 12-month follow-up and $44(50 \%)$ at 24-month follow-up. Participants with missing data at follow-up had on average higher levels of depressive symptoms at baseline on the MADRS-S (M=22.6, $\mathrm{SD}=8.6)$, compared with participants who provided follow-up data $(\mathrm{M}=16.8, \mathrm{SD}=8.7 ; \mathrm{t}(86)=-30$, $\mathrm{p}=0.003$ ) but did not differ significantly in BDD symptom severity, level of insight or in any other demographic characteristics $(p=0.08-0.85)$. Demographic and clinical characteristics of participants at baseline are shown in table 1.

\section{Primary outcome}

Figure 2 depicts the effect of treatment over time on the BDD-YBOCS. Mixed-effect models analysis showed that participants made significant improvements from baseline to post-treatment after receiving BDD-NET. The mean reduction from baseline to post-treatment on the BDD-YBOCS was $-8.23(95 \% \mathrm{CI}-9.92$ to $-6.54, \mathrm{p} \leq 0.001)$, with gains made from post-treatment to 3 months being stable; -0.43 (95\% CI -2.13 to $1.26, \mathrm{p}=0.610)$. From the 3-month follow-up to the 12 month follow-up, further improvements were observed, with an additional reduction on the BDD-YBOCS of -4.16 points (95\% CI -6.30 to $-2.01, \mathrm{p} \leq 0.001)$. These effects were sustained from the 12-month follow-up to the 24month follow-up $(-1.02$ (95\% CI -3.37 to $1.32, \mathrm{p}=0.382))$. Overall, the mean reduction on the BDD-YBOCS from baseline to the 24-month follow-up was -13.42 points (95\% CI -15.57 to $-11.27, \mathrm{p} \leq 0.001)$, corresponding to a large within-group effect size; $d=1.79$ (95\% CI 1.40 to 2.18), and on average a $52 \%$ reduction in symptoms. The estimated means of the primary and secondary outcomes are presented in table 2.

\section{Treatment response and remission}

At 24-month follow-up, 69\% (95\% CI 57\% to 80\%) were classified as responders $(\geq 30 \%$ reduction on the
BDD-YBOCS from baseline) and 57\% (95\% CI $45 \%$ to $69 \%$ ) of the participants were classified as much improved or very much improved on the Clinical Global Impressions Scale. In total, $56 \%$ (95\% CI $43 \%$ to $69 \%$ ) no longer met DSM- 5 criteria for BDD (they had remitted).

In order to determine the trajectories of change during the follow-up period, participant's responder status at post-treatment was compared with their responder status at 24-month follow-up. Maintained response was defined as being a responder at post-treatment and still classified as a responder at follow-up. Delayed response was defined as being a non-responder at post-treatment but classified as a responder at follow-up. Relapse was defined as being a responder at post-treatment but as a non-responder at follow-up, and non-response was defined as being a non-responder both at post-treatment and at follow-up. Most participants (40\%) maintained their classification as responders from post-treatment to the 24-month follow-up, and another $29 \%$ had a delayed response to treatment. Out of those that were classified as non-responders at post-treatment, $21 \%$ were still non-responders at follow-up, and $10 \%$ of those that were responders at post-treatment had relapsed at the 24-month follow-up. Figure 3 illustrates these trajectories. At 24-month follow-up, non-responders had on average higher scores on MADRS-S, were more likely to be diagnosed with depression and more likely to have had previous contact with psychiatric services (all $\mathrm{p}$ values $<0.05$ ).

\section{Secondary outcomes}

Depressive symptoms measured with the MADRS-S significantly decreased from baseline to 24-month follow-up with a mean reduction of -5.04 points ( $95 \%$ CI -5.94 to -1.99 , $\mathrm{p} \leq 0.001)$. The within-group effect size from baseline to 24-month follow-up was moderate, $d=0.62$ (95\% CI 0.25 to 0.99). Global functioning assessed with the GAF was higher at 24 months when compared with baseline GAF, with an average increase of 10.77 points $(95 \%$ CI 8.63 to $12.91, \mathrm{p} \leq 0.001)$, corresponding to a large effect size of $\mathrm{d}=1.47$ (95\% CI 1.09 to 1.84 ). Health-related quality of life measured with the EQ5D did not improve from baseline to post-treatment $(\mathrm{p}=0.393)$, but did improve from post-treatment to 3-month and 12-month follow-ups with a mean increase of 0.09 (95\% CI 0.03 to $0.15, \mathrm{p}=0.002$ ) and $0.11(95 \%$ CI 0.04 to $0.18, \mathrm{p}=0.001)$ points, respectively. However, at the 24-month follow-up, these gains were no longer significant, as the EQ5D score had slightly decreased ( $\mathrm{p}=0.062$ ). The baseline to 24-month follow-up within group effect size for the EQ5D was small, $d=0.29$ (95\% CI -0.07 to 0.65$)$.

\section{Participant's reasons for applying to the original trial}

At the 12-month follow-up, 49 of 54 participants that took part in the follow-up agreed to be briefly interviewed about reasons for applying to the original trial. Sixty-three per cent said that they would not have sought help for their BDD if it were not for the trial, $31 \%$ would have sought care regardless and $6 \%$ did not provide a clear answer. A 
Allocation (Enander et al. 2016)

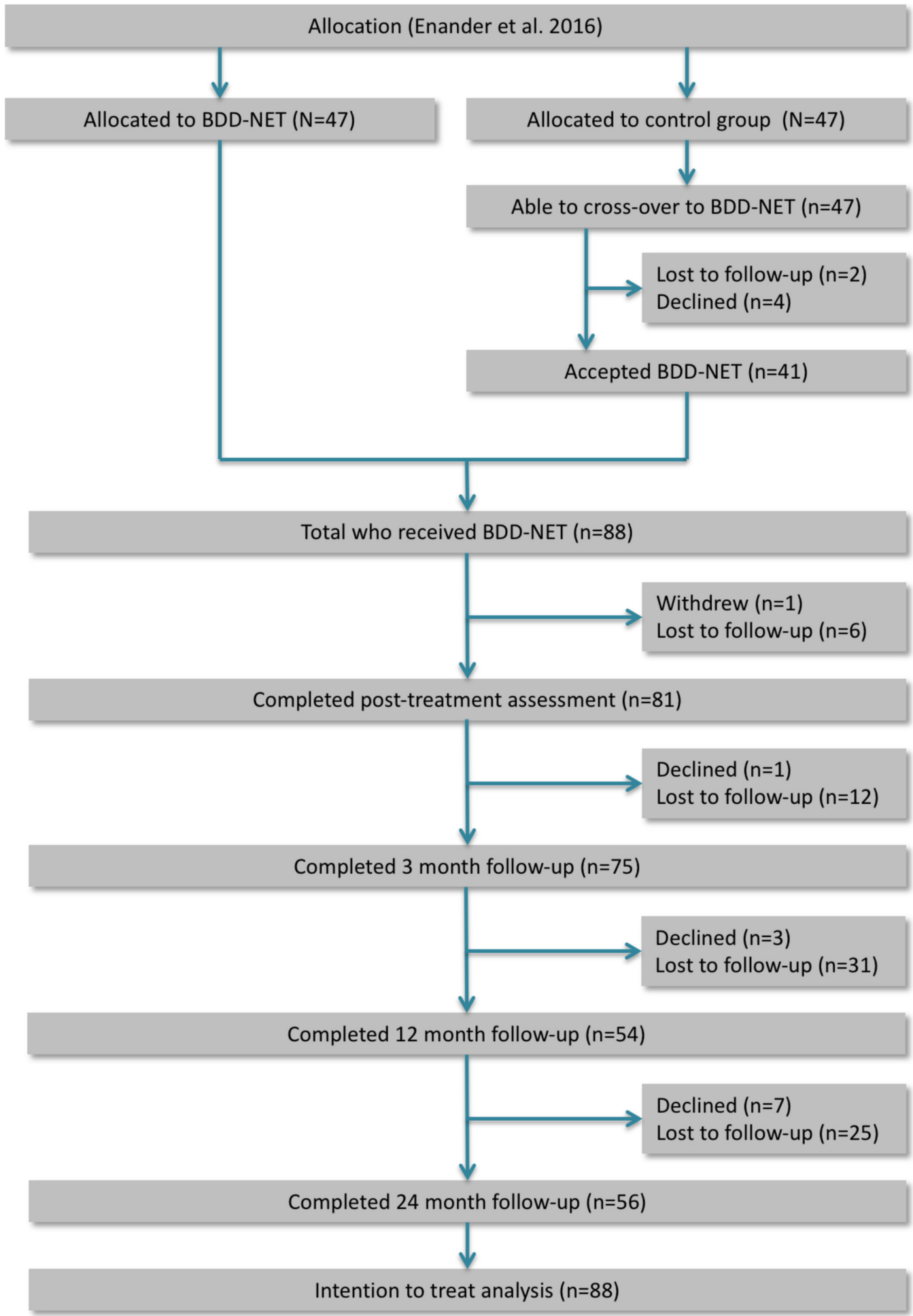

Figure 1 Flow of participants with body dysmorphic disorder (BDD) through the long-term study of therapist-guided internetbased cognitive-behavioural therapy (BDD-NET).

major reason for applying to the trial was the possibility to do the treatment from home without having to travel to a clinic, endorsed by $63 \%$ of participants. Furthermore, $44 \%$ reported that it was specifically the possibility to do the treatment online, without having to sit face-to-face with a clinician as an important factor for applying to the trial in the first place. Reasons disclosed by participants for not wanting to seek help for their BDD 'face-to-face' were in part due to feelings of shame and stigma about being perceived as vain. In total, $59 \%$ reported they felt that they had not been taken seriously when they previously had disclosed their appearance concerns to a health professional.

Additional treatment during the follow-up

Information about additional treatment in the period between post-treatment and 24-month follow-up was 
Table 1 Sociodemographic and clinical characteristics of the sample at baseline

\begin{tabular}{|c|c|}
\hline Variable & \\
\hline \multicolumn{2}{|l|}{ Gender, n (\%) } \\
\hline Women & $74(84)$ \\
\hline Men & $14(16)$ \\
\hline \multicolumn{2}{|l|}{ Age, mean (SD) } \\
\hline Mean age & $32.5(11.6)$ \\
\hline Min-max & $18-72$ \\
\hline Married, n (\%) & $14(16)$ \\
\hline \multicolumn{2}{|l|}{ Highest education, $\mathrm{n}(\%)$} \\
\hline Primary school & $9(10)$ \\
\hline High school & $51(58)$ \\
\hline College/university & $26(30)$ \\
\hline Doctorate & $2(2)$ \\
\hline \multicolumn{2}{|l|}{ Occupational status } \\
\hline Working & $49(56)$ \\
\hline Student & $22(25)$ \\
\hline Retired & $3(3)$ \\
\hline Unemployed & $13(15)$ \\
\hline Disability pension & $1(1)$ \\
\hline \multicolumn{2}{|l|}{ Insight, n (\%) } \\
\hline Good & $46(52)$ \\
\hline Poor & $34(39)$ \\
\hline Delusional & $8(9)$ \\
\hline \multicolumn{2}{|l|}{ BDD duration, mean (SD) } \\
\hline Mean length in years & $18.8(13.3)$ \\
\hline \multicolumn{2}{|l|}{ Comorbidity, n (\%) } \\
\hline Current depressive episode & $44(50)$ \\
\hline Panic disorder & $3(3)$ \\
\hline Social anxiety disorder & $25(28)$ \\
\hline Generalised anxiety disorder & $15(17)$ \\
\hline Bulimia nervosa & $2(2)$ \\
\hline ADHD & $2(2)$ \\
\hline Obsessive-compulsive disorder & $8(9)$ \\
\hline \multicolumn{2}{|l|}{ Current medication, $\mathrm{n}(\%)$} \\
\hline SSRI & $12(14)$ \\
\hline SNRI & $2(2)$ \\
\hline Other antidepressants & $7(8)$ \\
\hline Mood stabilisers & $4(5)$ \\
\hline Benzodiazepines & $3(3)$ \\
\hline Neuroleptics & $2(2)$ \\
\hline Methylphenidate & $2(2)$ \\
\hline \multicolumn{2}{|l|}{ Previous psychosocial treatment, $\mathrm{n}(\%)$} \\
\hline CBT for BDD & $10(11)$ \\
\hline Psychosocial treatment for depression or anxiety & $53(60)$ \\
\hline \multicolumn{2}{|l|}{ Plastic surgery, $\mathrm{n}(\%)$} \\
\hline Previous plastic surgery & $21(24)$ \\
\hline Mean no. of surgeries (SD) & $2.3(1.7)$ \\
\hline Min-max no. of surgeries & $1-6$ \\
\hline
\end{tabular}

$A D H D$, attention-deficit hyperactivity disorder; BDD, body dysmorphic disorder; CBT, cognitive-behavioural therapy; SNRI, serotoninnorepinephrine reuptake Inhibitors; SSRI, selective serotonin reuptake inhibitors.

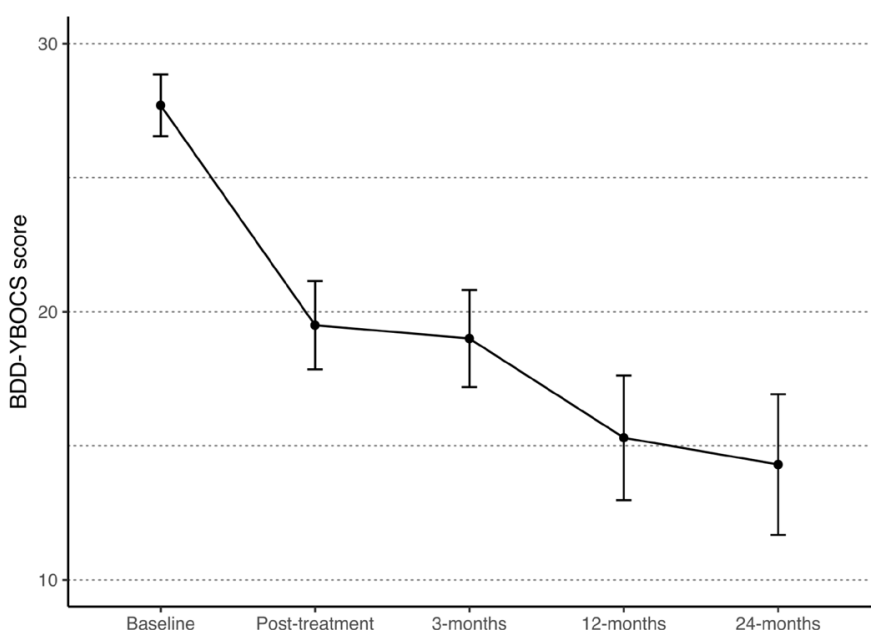

Figure 2 Effect of treatment over time on the Yale-Brown Obsessive Compulsive Scale modified for body dysmorphic disorder (BDD-YBOCS) with 95\% Cls.

provided by 71 of the participants. In total, 12/71 (17\%) reported that they had received additional care during the 2-year follow-up period. Treatments that had been provided were pharmacotherapy $(\mathrm{n}=11)$, additional faceto-face $\operatorname{CBT}(\mathrm{n}=7)$ and psychodynamic psychotherapy $(\mathrm{n}=1)$. One participant had undergone plastic surgery twice during the follow-up period. Of those who had sought additional care, 4/12 (33\%) had been non-responders at post-treatment and were still classified as non-responders at 24-month follow-up, 2/12 (17\%) were responders at post-treatment, before receiving any additional care and maintained their responder status at follow-up, 4/12 (33\%) had been non-responders at post-treatment but were responders at follow-up, and 2/12 (17\%) had been responders at post-treatment but were classified as non-responders at follow-up. Participants who had sought further treatment had on average higher scores on the BDD-YBOCS and MADRS-S and were more likely to be diagnosed with major depressive disorder at 24 months, compared with those who had not received any additional care (all $\mathrm{p}$ values $<0.05$ ).

\section{DISCUSSION}

The aim of this study was to investigate the long-term effectiveness of BDD-NET, a therapist-guided internet-based CBT programme for BDD by reassessing participants 2years after treatment. BDD symptom severity decreased after receiving BDD-NET, and patients continued improving during the long-term follow-up. Two years after treatment, $69 \%$ of participants were classified as responders, and $56 \%$ no longer met diagnostic criteria for BDD. Improvements on depressive symptoms and global functioning were also sustained long term but not health-related quality of life. A majority of participants reported that it was the possibility to do the treatment from home that made them seek help for their BDD in the first place. 
Table 2 Estimated means and mean differences comparing baseline to follow-ups using linear mixed models ( $\mathrm{n}=88$ )

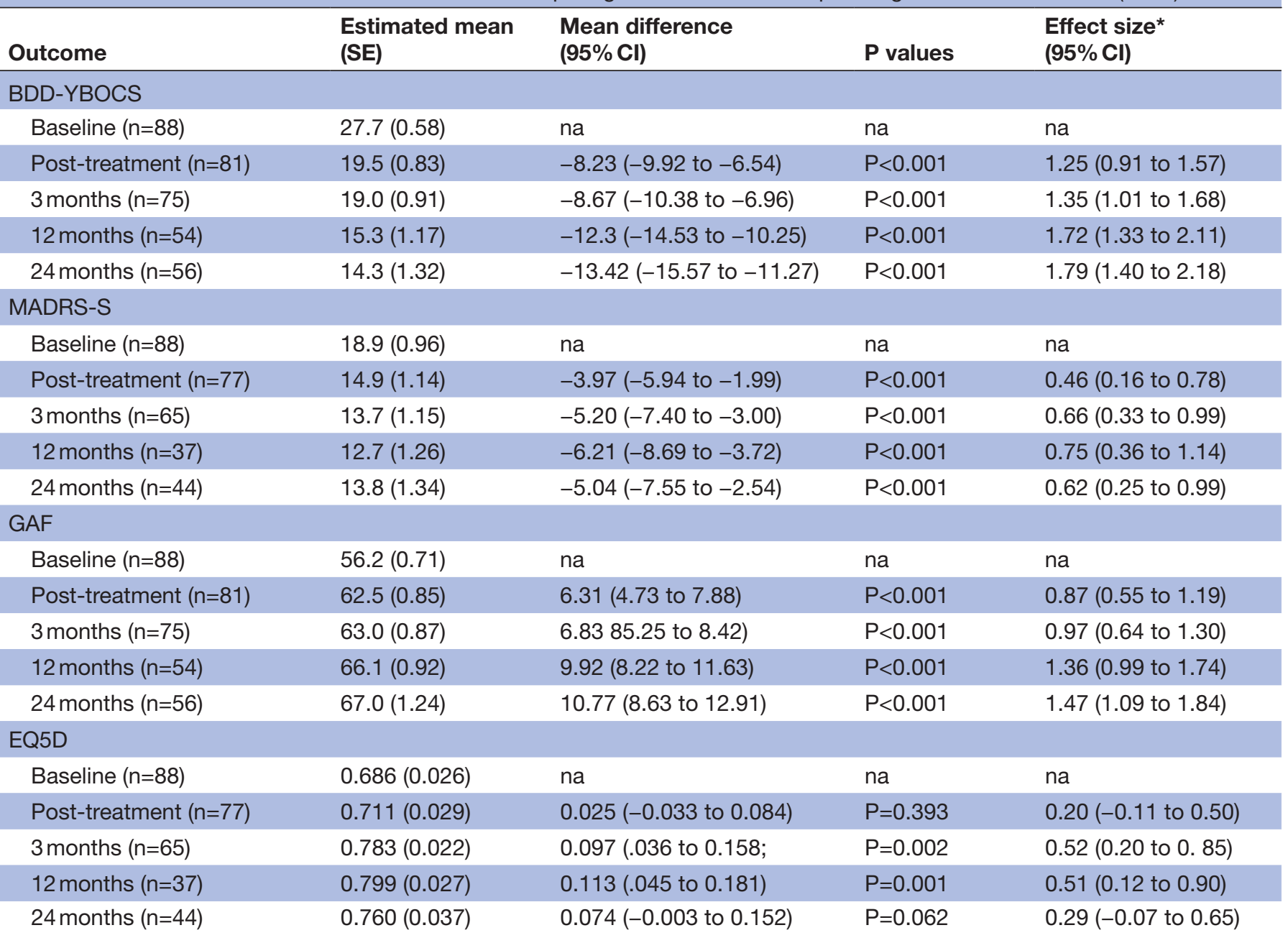

BDD-YBOCS, Yale-Brown obsessive compulsive scale modified for body dysmorphic disorder; EQ5D, EuroQol EQ5D; GAF, Global Assessment of Functioning; MADRS-S, Montgomery-Åsberg Depression Rating Scale Self-Rated; na, not applicable.

${ }^{*}$ Cohen's d effect sizes between groups calculated from observed data.

The long-term effectiveness of BDD-NET is in line with the previous three studies published of the long-term outcomes of CBT for BDD. ${ }^{13-15}$ In the current study, participants continued to improve on the BDD-YBOCS during the follow-up, and $29 \%$ had a delayed response to treatment. In BDD-NET, the therapist only provides minimal support, and participants have to take greater responsibility for completing tasks and reaching goals. This may translate to an increased self-efficacy. ${ }^{39}$ Perhaps participants continued to employ the techniques that they had acquired during the acute treatment phase in their daily lives, in part explaining the additional improvements seen in BDD symptoms during the follow-up. Health-related quality of life measured with the EQ5D was significantly improved from baseline to the 12-month follow-up but did slightly decrease between the 12-month and 24-month follow-ups so that the improvement no longer was significant $(p=0.06)$. EQ5D is primarily designed to assess quality of life in patients with somatic illnesses. It includes questions about anxiety/depression and mobility, pain and self-care, none of which are usually impaired in BDD. Considering this, EQ5D may not be the ideal instrument for assessing quality of life in BDD. For example, it has been found to be somewhat sensitive to change in patients with milder symptoms of depression/ anxiety, ${ }^{40}$ but it has also been shown to be less sensitive to change in more severe psychiatric patients. ${ }^{41}$ However, it is widely used in health economic evaluations to calculate quality-adjusted life years, allowing for future cost-effectiveness studies of BDD-NET.

In the first study ever published on the long-term outcomes of CBT for BDD, 10 participants were randomised to exposure and response prevention with or without a 6 -month maintenance programme. ${ }^{13}$ Treatment gains were sustained for 2 years, and adding booster sessions during the follow-up period seemed to provide some beneficial effect, although the study was underpowered to be able to detect any differences between the two groups. Evidence suggests that booster sessions in CBT for depression and anxiety may generate better outcomes and more sustained effects, compared with CBT without booster sessions. ${ }^{42}$ Using the BDD-NET platform to deliver 


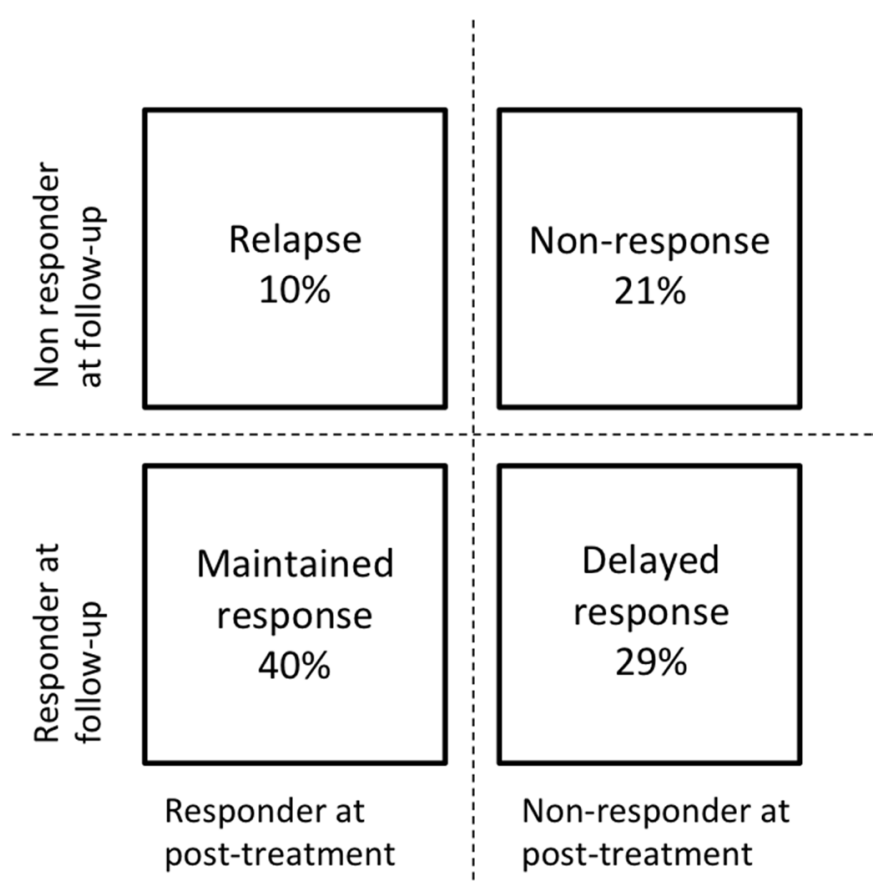

Figure 3 Trajectories of participants' treatment response status from post-treatment to 24-month follow-up.

a maintenance programme after termination of CBT may be ideal, as all communication within the platform is asynchronous, and participants could easily contact their therapist without having to travel to a clinic. This has been explored in a related condition, obsessive-compulsive disorder, where participants were randomised to either an internet-based booster programme or no booster after 12 weeks of treatment. Results showed that participants who were randomised to receive additional booster sessions had had fewer relapses at the 2-year follow-up. ${ }^{43}$ Adding an internet-based booster programme may potentially help decrease relapse and improve long-term outcomes in CBT for BDD, an approach that warrants further research.

The proportion of responders in our sample $(69 \%)$ is somewhat higher when compared with the two recent studies published on the long-term outcomes of CBT for BDD in adults $(46 \%)^{14}$ and adolescents $(50 \%) .{ }^{15}$ However, the participants in our sample were self-referred, whereas most of the participants in the adult and adolescent samples had been referred to a tertiary care setting for specialised treatment. Clearly, internet-based CBT should not be seen as a substitute for face-to-face CBT, but rather as a way to increase access to CBT for mild to moderate cases with low suicide risk, and may be particularly useful in a stepped-care approach. ${ }^{23}{ }^{24}$ The long-term efficacy of BDD-NET suggests that it could be disseminated to health services; however, it will be important to further understand for whom internet-based CBT is most suitable. Future research should strive to identify reliable predictors and moderators that can be used to personalise treatment selection, and thus reduce the risk of unnecessary treatment failures.
Shame and fear of stigma are important barriers for many people with BDD to seek and engage in treatment for their appearance concerns. ${ }^{17} 18$ Forty-four per cent of the trial participants said that it was the possibility to do the treatment online, without having to meet face-to-face with a clinician that made them seek help for their BDD in the first place. Thus, internet-based CBT has the potential to lower the threshold for BDD sufferers to engage in treatment. Furthermore, patients who seek help from health services often do not get access to CBT due to a lack of trained therapists. ${ }^{16}$ Internet-based CBT has the potential to improve access to evidence-based care, and it eliminates geographical barriers as patients can participate in the treatment from the comfort of their home and without having to schedule appointments. The treatment is also delivered online as a series of modules (eg, self-help texts and instructions on how to do exposure and response prevention) ensuring that all patients get exactly the same treatment, eliminating any potential therapist drift and deviation from treatment protocols that may occur in face-to-face therapy. ${ }^{44}$ Another advantage of internet-based CBT is that all communication between participant and therapist is asynchronous. Participants can work with the modules on evenings and weekends, when they have the most time, and when the therapist gets back in the office the following morning, he or she can answer any questions that the participant may have sent, provide feedback on completed homework assignments and send supportive messages during regular clinic hours.

There are several limitations to take into consideration when interpreting the results. Participants were self-referred and had on average fair insight; thus, the results may not be generalisable to all people with BDD. For ethical reasons, there was no untreated group to compare longterm follow-up outcomes with. This limits the possibility to make causal interference of what caused the further improvements on the BDD-YBOCS during the follow-up. Also, due to the nature of the trial, assessors were not blinded at the 1-year and 2-year follow-up. Participants lost to follow-up at 1 year and 2 years after treatment was $61 \%$ and $64 \%$, respectively. A sensitivity analysis showed that participants lost to follow-up, on average, had higher levels of depressive symptoms at baseline, which may have biased our results. Because of the relatively large amount of missing data, we carefully imputed the missing data using multiple imputations. In data simulation models, multiple imputations have been shown to perform well even with $50 \%$ of missing observations and may reduce bias compared with other strategies to handle missing data. ${ }^{37} 38$ Compared with the complete case analysis (data not shown), the analysis using multiple imputations resulted in more conservative treatment effects. However, the estimates should be interpreted with caution.

Seventeen per cent of the participants had received further treatment. Of those, four had made further improvements, but the majority of participants did not seek any further treatment and still continued to improve 
during the follow-up. This could have been due to the passage of time; however, we find it unlikely that the significant improvements observed could solely be explained by spontaneous recovery. Finally, participants were asked post hoc about their motives for applying to the trial at the 12-month follow-up, and this may have introduced recall bias.

\section{CONCLUSIONS}

BDD-NET is effective in the long term and has the potential to increase access to CBT for people with BDD. BDD-NET might also lower the threshold for sufferers of BDD to seek help in the first place. BDD-NET may be especially useful in a stepped-care approach, where mild to moderate cases can be offered BDD-NET, potentially freeing resources for more complex patients. Since we do not yet know for whom internet-based CBT is most suitable, the identification of reliable predictors and moderators that can be used to personalise treatment selection could be useful in choosing the right treatment from the start, and thus avoiding unnecessary treatment failures. A stepped-care non-inferiority controlled trial comparing BDD-NET to face-to-face CBT is warranted.

Contributors JE and CR had the original idea for the study and, with DM-C and BL, designed the trial variables and obtained the funding. JE and CR were responsible for study supervision. All authors were responsible for the acquisition of the data. $\mathrm{JE}$ and $\mathrm{BL}$ carried out the statistical analysis. JE drafted the manuscript, which was revised by BL, LA, MR, OF, OC, EA, SD, LL, VZI, DM-C and CR. All researchers were independent of the funders.

Funding This study was funded through the regional agreement on medical training and clinical research (ALF) between the Stockholm County Council and Karolinska Institutet, the Swedish Research Council (grant No: K2013-61X-22168-01-3) and the Swedish Society of Medicine (Söderströmska Königska sjukhemmet, grant no: SLS3B4451).

Disclaimer The funders had no part in the study design, in the collection, analysis and interpretation of data, in the writing of the report or in the decision to submit the article for publication.

Competing interests None declared.

Patient consent Obtained.

Ethics approval The authors assert that all procedures contributing to this work comply with the ethical standards of the relevant national and institutional committees on human experimentation and with the Helsinki Declaration of 1975 , as revised in 2008. The regional ethical review board in Stockholm approved the study (registration ID: 2013/1773-31/4).

Provenance and peer review Not commissioned; externally peer reviewed.

Data sharing statement № additional data are available.

Open access This is an open access article distributed in accordance with the Creative Commons Attribution Non Commercial (CC BY-NC 4.0) license, which permits others to distribute, remix, adapt, build upon this work non-commercially, and license their derivative works on different terms, provided the original work is properly cited, appropriate credit is given, any changes made indicated, and the use is non-commercial. See: http://creativecommons.org/licenses/by-nc/4.0/.

\section{REFERENCES}

1. American Psychiatric Association. The diagnostic and statistical manual of mental disorders. 5th edn. Arlington, VA: American Psychiatric Publishing, 2013.
2. Grant JE, Kim SW, Crow SJ. Prevalence and clinical features of body dysmorphic disorder in adolescent and adult psychiatric inpatients. $J$ Clin Psychiatry 2001;62:517-22.

3. Veale D, Gledhill LJ, Christodoulou P, et al. Body dysmorphic disorder in different settings: A systematic review and estimated weighted prevalence. Body Image 2016;18:168-86.

4. Bjornsson AS, Didie ER, Grant JE, et al. Age at onset and clinical correlates in body dysmorphic disorder. Compr Psychiatry 2013;54:893-903.

5. Enander J, Ivanov VZ, Mataix-Cols D, et al. Prevalence and heritability of body dysmorphic symptoms in adolescents and young adults: a population-based nationwide twin study. Psychol Med 2018;40:1-8.

6. Phillips KA, Menard W, Quinn E, et al. A 4-year prospective observational follow-up study of course and predictors of course in body dysmorphic disorder. Psychol Med 2013;43:1109-17.

7. Phillips KA, Menard W, Fay C, et al. Psychosocial functioning and quality of life in body dysmorphic disorder. Compr Psychiatry 2005;46:254-60.

8. Phillips KA, Menard W. Suicidality in body dysmorphic disorder: a prospective study. Am J Psychiatry 2006;163:1280-2.

9. National Collaborating Centre for Mental Health (UK). Obsessivecompulsive disorder: core interventions in the treatment of obsessive-compulsive disorder and body dysmorphic disorder. Leicester (UK): British Psychological Society, 2006.

10. Angermeyer MC, Matschinger $\mathrm{H}$, Schomerus $\mathrm{G}$. Attitudes towards psychiatric treatment and people with mental illness: changes over two decades. Br J Psychiatry 2013;203:146-51.

11. Ipser JC, Sander C, Stein DJ. Pharmacotherapy and psychotherapy for body dysmorphic disorder. Cochrane Database Syst Rev 2009;1:CD005332.

12. Harrison A, Fernández de la Cruz L, Enander J, et al. Cognitivebehavioral therapy for body dysmorphic disorder: a systematic review and meta-analysis of randomized controlled trials. Clin Psychol Rev 2016;48:43-51.

13. McKay D. Two-year follow-up of behavioral treatment and maintenance for body dysmorphic disorder. Behav Modif 1999;23:620-9

14. Veale D, Miles S, Anson M. Long-term outcome of cognitive behavior therapy for body dysmorphic disorder: a naturalistic case series of 1 to 4 years after a controlled trial. Behav Ther 2015;46:775-85.

15. Krebs G, de la Cruz LF, Monzani B, et al. Long-term outcomes of cognitive-behavioral therapy for adolescent body dysmorphic disorder. Behav Ther 2017;48:462-73.

16. Cavanagh K. Geographic inequity in the availability of cognitive behavioural therapy in England and Wales: a 10-year update. Behav Cogn Psychother 2014;42:497-501.

17. Marques L, Weingarden HM, Leblanc NJ, et al. Treatment utilization and barriers to treatment engagement among people with body dysmorphic symptoms. J Psychosom Res 2011;70:286-93.

18. Buhlmann $U$. Treatment barriers for individuals with body dysmorphic disorder: an internet survey. J Nerv Ment Dis 2011;199:268-71.

19. Phillips KA, Pagano ME, Menard W, et al. A 12-month follow-up study of the course of body dysmorphic disorder. Am J Psychiatry 2006;163:907-12.

20. Hedman E, Ljótsson B, Lindefors N. Cognitive behavior therapy via the Internet: a systematic review of applications, clinical efficacy and cost-effectiveness. Expert Rev Pharmacoecon Outcomes Res 2012;12:745-64.

21. Hedman E, Andersson E, Ljótsson B, et al. Cost effectiveness of internet-based cognitive behaviour therapy and behavioural stress management for severe health anxiety. BMJ Open 2016;6:e009327.

22. Andersson $\mathrm{E}$, Hedman $\mathrm{E}$, Ljótsson $\mathrm{B}$, et al. Cost-effectiveness of internet-based cognitive behavior therapy for obsessive-compulsive disorder: results from a randomized controlled trial. J Obsessive Compuls Relat Disord 2015;4:47-53.

23. Enander J, Ivanov VZ, Andersson E, et al. Therapist-guided, Internetbased cognitive-behavioural therapy for body dysmorphic disorder (BDD-NET): a feasibility study. BMJ Open 2014;4:e005923-3.

24. Enander J, Andersson E, Mataix-Cols D, et al. Therapist guided internet based cognitive behavioural therapy for body dysmorphic disorder: single blind randomised controlled trial. BMJ 2016;352:i241.

25. Phillips KA, Hollander E, Rasmussen SA, et al. A severity rating scale for body dysmorphic disorder: development, reliability, and validity of a modified version of the Yale-Brown Obsessive Compulsive Scale. Psychopharmacol Bull 1997;33:17-22.

26. Rohde P, Lewinsohn PM, Seeley JR. Comparability of telephone and face-to-face interviews in assessing axis I and II disorders. Am J Psychiatry 1997;154:1593-8. 
27. Sheehan DV, Lecrubier $\mathrm{Y}$, Sheehan $\mathrm{KH}$, et al. The Mini-International Neuropsychiatric Interview (M.I.N.I.): the development and validation of a structured diagnostic psychiatric interview for DSM-IV and ICD10. J Clin Psychiatry 1998:59(Suppl 20):22-33. quiz34-57.

28. Phillips KA, Hart AS, Menard K. Psychometric evaluation of the YaleBrown Obsessive-Compulsive Scale Modified for Body Dysmorphic Disorder (BDD-YBOCS). J Compuls Relat Disord 2014;3:1-4.

29. Svanborg P, Asberg M. A new self-rating scale for depression and anxiety states based on the Comprehensive Psychopathological Rating Scale. Acta Psychiatr Scand 1994;89:21-8.

30. American Psychiatric Association. Diagnostic and statistical manual of mental disorders: Dsm-iv-tr. Washington, DC: American Psychiatric Association, 2000.

31. EuroQol Group. EuroQol--a new facility for the measurement of health-related quality of life. Health Policy 1990;16:199-208.

32. Verbeke G, Molenberghs G. Linear mixed models for longitudinal data: Springer Science \& Business Media, 2009.

33. White IR, Royston P, Wood AM. Multiple imputation using chained equations: Issues and guidance for practice. Stat Med 2011;30:377-99.

34. Hardt J, Herke M, Leonhart R. Auxiliary variables in multiple imputation in regression with missing $X$ : a warning against including too many in small sample research. BMC Med Res Methodol 2012;12:184

35. Rubin DB, Schenker N. Multiple imputation in health-care databases: an overview and some applications. Stat Med 1991;10:585-98.
36. Sterne JA, White IR, Carlin JB, et al. Multiple imputation for missing data in epidemiological and clinical research: potential and pitfalls. BMJ 2009;338:b2393.

37. Soullier N, de La Rochebrochard E, Bouyer J. Multiple imputation for estimation of an occurrence rate in cohorts with attrition and discrete follow-up time points: a simulation study. BMC Med Res Methodol 2010;10:79

38. Allison PD. Missing data. Thousand Oaks: Sage Publications, 2002.

39. Bandura A. Self-efficacy: toward a unifying theory of behavioral change. Psychol Rev 1977;84:191-215.

40. Lamers LM, Bouwmans CA, van Straten A, et al. Comparison of EQ-5D and SF-6D utilities in mental health patients. Health Econ 2006;15:1229-36.

41. van de Willige G, Wiersma D, Nienhuis FJ, et al. Changes in quality of life in chronic psychiatric patients: a comparison between EuroQo (EQ-5D) and WHOQoL. Qual Life Res 2005;14:441-51.

42. Gearing RE, Schwalbe CS, Lee R, et al. The effectiveness of booster sessions in CBT treatment for child and adolescent mood and anxiety disorders. Depress Anxiety 2013;30:800-8.

43. Andersson E, Steneby S, Karlsson K, et al. Long-term efficacy of Internet-based cognitive behavior therapy for obsessive-compulsive disorder with or without booster: a randomized controlled trial. Psychol Med 2014;44:2877-87.

44. Waller G. Evidence-based treatment and therapist drift. Behav Res Ther 2009;47:119-27. 